"This is the peer reviewed version of the following article: McMillan H, Westerberg I, Branger F. Five guidelines for selecting hydrological signatures. Hydrological Processes. 2017; 1-5., which has been published in final form at https://doi.org/10.1002/hyp.11300. This article may be used for non-commercial purposes in accordance with Wiley Terms and Conditions for Self-Archiving."

\title{
Five Guidelines for Selecting Hydrological Signatures
}

\author{
Hilary McMillan*1, Ida Westerberg ${ }^{2}$, Flora Branger ${ }^{3}$ \\ ${ }^{1}$ Department of Geography, San Diego State University, USA \\ ${ }^{2}$ IVL Swedish Environmental Research Institute, Stockholm, Sweden \\ ${ }^{3}$ IRSTEA, UR HHLY, Hydrology-Hydraulic Department, Centre de Lyon-Villeurbanne, \\ France \\ *hmcmillan@sdsu.edu
}

\section{Introduction}

Hydrological signatures are index values derived from observed or modelled series of hydrological data such as rainfall, flow or soil moisture. They are designed to extract relevant information about hydrological behaviour, such as to identify dominant processes, and to determine the strength, speed and spatiotemporal variability of the rainfall-runoff response.

A signature is often scalar; common examples of signatures calculated from streamflow include mean flow, baseflow index or slope of the Flow Duration Curve (FDC). Signature indices have long been used in hydrological analyses, but the concept of a hydrological signature was first explicitly described by Gupta et al. (2008) in the context of deriving minimal representations of the relevant information contained in a hydrological dataset.

Hydrological signatures have many applications, for example: learning about hydrological processes in a given location, comparing observed hydrological behaviour against theoretical or modelled behaviour, and determining hydrological similarity across time or space. However, the link between signature values and hydrological behaviour and processes is not always straightforward, leading to uncertainty and variability in hydrologists' signature choices.

Most studies use a selection of different signatures to capture different aspects of the catchment response, for example evaluating overall flow distribution as well as high and low flow extremes and flow timing. Such studies may choose their own signatures, or borrow subsets of signatures used in other works. There is little agreement regarding guidelines or requirements for successful choices of signatures or sets of signatures. We believe that reaching a consensus on selection criteria for hydrological signatures will assist hydrologists 
in choosing between competing signatures and facilitate comparison between hydrological studies.

The aim of this paper is to encourage a more rigorous approach to hydrological signature selection, which considers the ability of signatures to represent hydrological behaviour and underlying processes for the catchment and application in question. To this end, we propose a set of guidelines for selecting hydrological signatures. We describe five criteria that any hydrological signature should conform to: Identifiability, Robustness, Consistency, Representativeness, and Discriminatory Power. We explain why each criterion is important and give examples of design or adaption of signatures to meet the guidelines.

\section{Signature Selection Review}

Any study using hydrological signatures requires the hydrologist to choose one or more appropriate signatures. The reasons given by authors (including ourselves!) are varied, but typically belong to one of the following categories.

(1) A set or subset of those signatures used in a previous study; for example: "signatures utilised in previous studies” (Coxon, Freer, Wagener, Odoni, \& Clark, 2014, p. 6139).

(2) Signatures designed to capture a broad range of hydrological information. For example: "signature information of interest for a wide range of applications and [...] across a range of flow behaviour" (Westerberg et al., 2016, p. 1850), "to capture independent information about catchment behaviour at annual, seasonal and daily timescales, and to capture average as well as extreme hydrological behaviour" (Sawicz et al., 2014, p. 274), "constructed to reflect different aspects of the hydrograph" (Euser et al., 2013, p. 1896).

(3) A set of signatures that are independent or uncorrelated from one another; for example: "the response characteristics [were] checked for redundancy" (Yadav, Wagener, \& Gupta, 2007, p. 1762)

(4) Signatures that can be related to hydrological function; for example: "those [...] that have an interpretable link to catchment function” (Sawicz, Wagener, Sivapalan, Troch, \& Carrillo, 2011, p. 2897), "signature patterns of behaviour that are related to the primary watershed functions" and "capable of extracting useful information pertaining to system functioning" (Yilmaz, Gupta, \& Wagener, 2008, p. 7).

We note that in several other studies we reviewed, hydrological signature choices were simply stated with little or no reasons given for the choice.

\section{Guidelines for Selecting Hydrological Signatures}

We argue that the design or choice of any signature should be carefully evaluated in terms of its ability to serve the study aim, such as understanding dominant upstream processes or diagnostic model evaluation. Therefore, we propose a set of five guidelines outlined below, and suggest that an acceptable signature should satisfy all or most of these. If choosing between two competing signatures that seek to describe the same process or characteristic, the guidelines will promote a rigorous comparison. Signature users may need to revise such guidelines depending on the main purpose of their analysis, but we believe that a structured evaluation of this type will lead to more robust outcomes.

(1) Identifiability. Uncertainty in the signature value should be small when compared to the range of values between different catchments, ensuring that the uncertainty does not obscure inter-catchment differences (Westerberg et al., 2016). An uncertainty estimate should be cited alongside all signature values, whether calculated from the data or estimated from the 
literature. Signatures can sometimes be designed to be robust to known data uncertainties (Westerberg \& McMillan, 2015).

(2) Robustness. Signature values should be independent of the peculiarities of the data collection design. This includes instrument type, exact measurement procedure, length of time series, dates of record, etc. The robustness criterion ensures that the signature describes the catchment itself rather than the measurement of the catchment. For example, this criterion is highly relevant to soil moisture signatures, which should ideally be independent of soil moisture probe type and quirks of installation.

(3) Consistency. Signature values should be comparable across catchments, and insensitive to irrelevant factors. This characteristic can frequently be incorporated into the signature design, such as a normalisation by catchment area, to maximise the usefulness of the signature in comparing catchments according to underlying hydrological function rather than superficial catchment characteristics.

(4) Representativeness. Hydrological properties are frequently heterogeneous at small scales, whereas modelling or management applications require estimates of emergent, catchment-scale behaviour. Therefore signatures should describe average (sub-)catchment behaviour where this is meaningful. Signatures based on local properties (e.g. soil moisture or water table level) should preferably not be sensitive to the exact location of the sensor below the (sub-)catchment scale. Signatures based on river flow (e.g. flow dynamics or chemical properties) should represent average upstream tributary behaviour, or users should be aware of the limitation if this is not the case. This implies that signature values should vary approximately linearly with the underlying descriptor, for example, as described by Kirchner (2016a, 2016b) for signatures that use annual isotope cycle amplitude to describe water transit time. Similar concerns apply to signatures describing flood peaks as they merge along the river network.

(5) Discriminatory power. This criterion specifies that the knowledge of signature values should increase our knowledge of hydrological function. Therefore, differences in signature values should increase with differences in hydrological function. Catchments which the hydrologist identifies as functionally similar should return similar signature values. Discriminatory power is also used in a modelling-specific sense, such that a constraint on the signature must reduce the prediction range of feasible models (Schaefli, 2016). Yilmaz et al. (2008) further required that a signature relates only to a subset of model parameters; they perturbed each parameter in turn to test the signature sensitivity.

\section{An example of signature design}

In this section, we describe an example of the design process for a signature, assessing possible signature designs against the guidelines above. Due to their ubiquity, we chose to consider a signature related to the Flow Duration Curve, selecting the FDC mid-section slope. The majority of hydrological studies using signatures include some aspects of the FDC, to characterize the magnitude and duration of observed flows, and "to evaluate the overall behaviour of a catchment” (Euser et al., 2013, p. 1899). As described by Yilmaz et al. (2008, p. 8), the FDC in general, and specifically the mid-section slope, can be related to catchment function as it is sensitive to vertical redistribution of soil water, i.e. the partitioning of water between quick flow and slow flow.

Using the slope of the FDC mid-section in linear or log space is common; for example in the range 0.2 to 0.7 (Yilmaz et al, 2008) or 0.33 to 0.66 (Yadav et al, 2007). Alternatively, the absolute values of the flow duration curve at a defined set of exceedance probabilities may be 
used (Westerberg et al., 2011) or a generalisation using the Nash-Sutcliffe Efficiency fit between the two FDCs of interest (Euser et al., 2013).

For this example, we consider FDCs, and specifically the FDC mid-section slope, as a proposed signature to quantify catchment overall behaviour and flashiness and evaluate it against each guideline. Specifically, we used the slope in the interval 0.33 to 0.66 , in log space, normalised by median flow. Where we find deficiencies, we suggest alternative signatures that may better conform to the guidelines.

(1) Identifiability. FDCs aggregate streamflow values over time, reducing their sensitivity to one-off errors (e.g. a blocked or malfunctioning flow gauge) and therefore improving the identifiability of the hydrological behaviour described by the FDC. However, because FDCs summarise streamflow, they are sensitive to errors in the rating curve function used to derive flow from a measured stage series. These errors can be significant, particularly for floods and low flows (McMillan \& Westerberg, 2015), and errors in slope of the rating curve translate directly into errors in slope of the FDC. By using the 0.33-0.66 range only, this signature will have lower uncertainty than those that include the extremes of the FDC. Westerberg et al. (2016) found that high uncertainties are also linked to uncertainty in rating curve breakpoints, and that FDC slope uncertainty was high compared to inter-catchment variability, leading to poor identifiability of inter-catchment differences.

(2) Robustness. FDCs are thought to represent stable characteristics of a catchment, and might therefore be expected to be robust to the length of time series or period of record. However, as demonstrated by Vogel and Fennessey (1994), even 30-year FDCs can be sensitive to the period of record, for example depending on whether any prolonged drought occurred during the period. They therefore recommend using the average annual FDC rather than the total FDC. The midsection slope signature is more robust to the period of record than the low-flow section of the FDC (e.g. exceedance probability $>0.7$; see Vogel and Fennessey's Fig 6). However, using the midsection slope of the average annual FDC could still be considered in drought-prone areas or if this signature is to be used in combination with low-flow signatures.

(3) Consistency. FDCs are often normalised by mean flow, median flow or catchment area. Multiplicative normalisations do not change the slope of the FDC in log space; however, they are relevant where absolute values of the FDC are compared. Whether or not scaling is recommended depends on the application. If the signature is used for model calibration, scaling by mean or median flow is not recommended as it would obscure bias in flow volumes. However, if the signature is used to compare water partitioning between quick/slow flow in different size catchments, scaling will be necessary.

(4) Representativeness. If we use the mid-section FDC slope as a signature to characterise catchment processes (e.g. flashiness, vertical re-distribution of water); we should consider whether the signature does or should represent an average of these process characteristics in the upstream tributaries. For example, suppose two tributaries meet, of equal annual flow volume, but one is dominated by ephemeral quick flow ("Flashy"), and the other by slow flow ("Not Flashy"). This example is illustrated in Figure 1 using a synthetic example based on two tributaries that each exhibit exponential recession behaviour, but with different time constants.

As shown in Figure 1b, the midsection slope (33\% to 66\%) of the confluence FDC is low, identical to that of the slow tributary, and is not influenced by the fast tributary. Therefore, this signature would characterise the entire catchment as "Not Flashy", even though half the flow volume comes from a "Flashy" catchment. This result derives directly from the 
definition of our signature, which only characterises flow at middle range values, after the flashy portion of the hydrograph has already passed. Care is therefore needed in interpreting the signature value, which represents accumulated flow behaviour rather than average upstream behaviour. The signature could be used in conjunction with other signatures targeting the high flow portion of the FDC, as is commonly done (e.g. Yilmaz et al., 2008).

Furthermore, we advise consideration of catchment-specific factors that can impact on hydrograph shape and therefore FDC signature values and their link to runoff generation processes. These include channel-based changes in the hydrograph shape not related to upstream averaging including diffusion of the flood wave, joining of tributaries with different flood timing, and heterogeneous influx of groundwater along the river network.
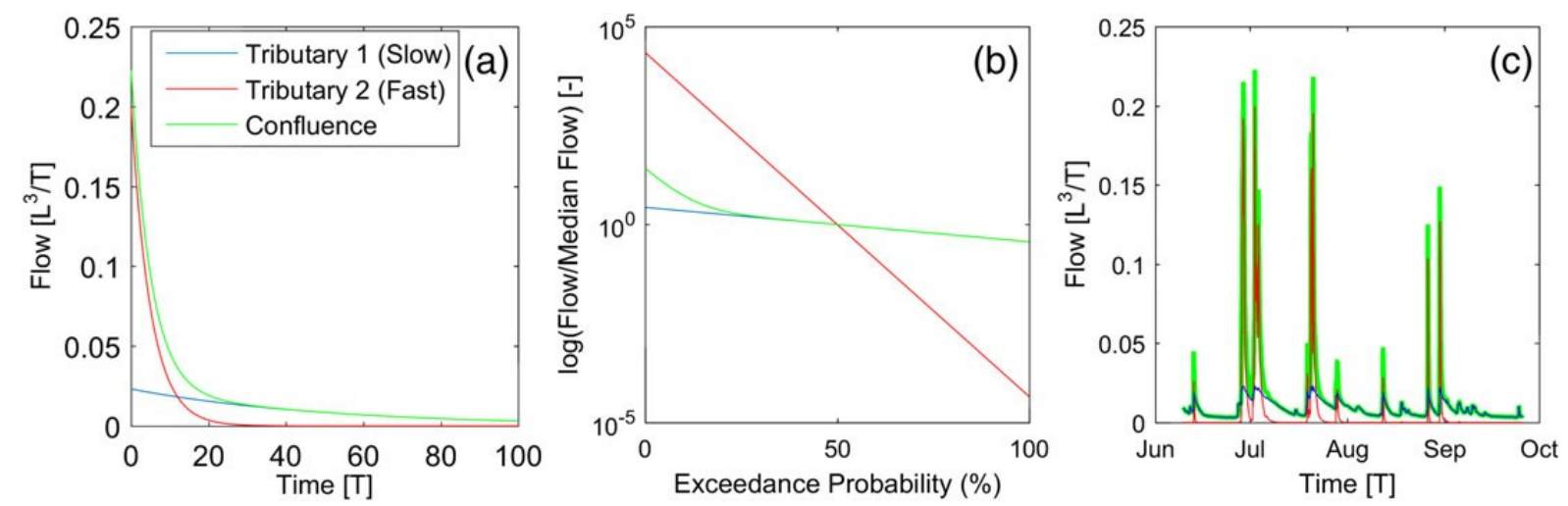

Figure 1: Example of the merging of two tributaries with different recession behaviours. (a) Characteristic recession curves for each tributary and their combination, (b) flow duration curves for the same rivers, normalised by median flow, and (c) Example flow series for each river, generated bycumulative distribution function (CDF)-correction of an arbitrary flow record.

(5) Discriminatory power. Using a similar synthetic example as above, we can see that two equal volume rivers with the same FDC midsection slope signature value can have visually very different flow behaviour, especially for high flows (Figure 2). These two rivers would be characterised as having identical flashiness/vertical water distribution if using only the midsection slope signature. Hence, the signature comparison may disagree with expert opinion on the similarity of the rivers. We may therefore need to revise our initial concept of which types of catchment behaviour are quantified by the FDC midsection slope, for example by explicitly distinguishing between surface and subsurface FDCs (e.g. Yokoo and Sivapalan, 2011), or by using this signature in combination with a high-flow FDC signature as suggested above. 


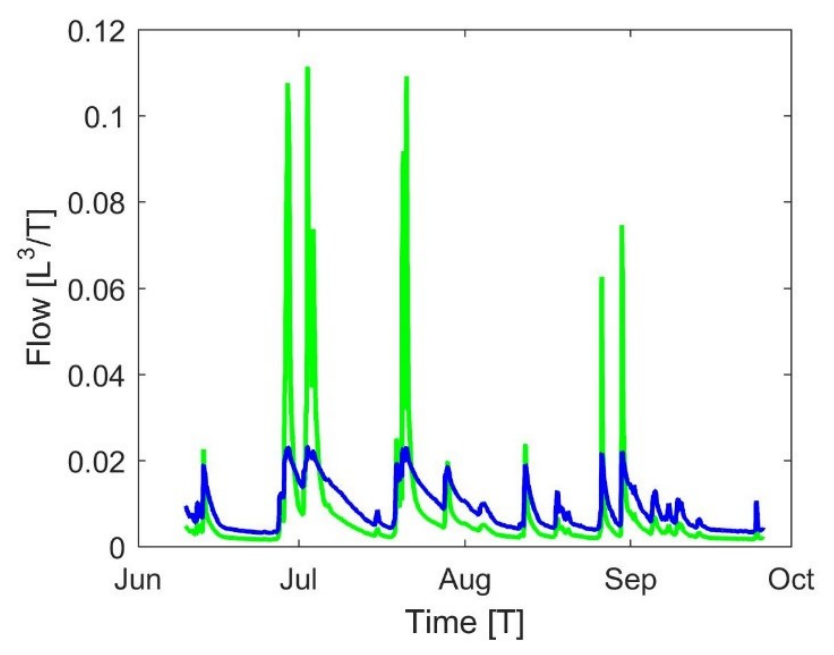

Figure 2: Example flow series of two rivers with identical flow volumes and flow duration curve midsection slope (interval 0.33 to 0.66 , in log space, normalised by median flow).

Synthesis. We found that the commonly used midsection slope of the FDC is, under some circumstances, limited in its ability to represent average upstream behaviour (representativeness) or to distinguish between visually different flow series (discriminatory power). While the issues are likely to be less severe in real catchments with more complex flow behaviour, this example demonstrates that this signature does not provide an overall picture of flashiness and vertical water distribution throughout the upstream catchment. Instead, it should be carefully interpreted as representing accumulated behaviour only during the mid-range flows/mid-recession period, and should be used alongside other signatures that target the fast-flow components of the catchment response.

\section{Discussion and Conclusions}

The aim of this commentary is to draw the attention of the hydrological community to the importance of signature choice, and the current lack of consensus as to preferred evaluation criteria for signatures. We would welcome discussion of how best to select hydrological signatures, setting the benefits of careful and consistent signature choice against the wide variety of signature applications.

The concept of "discriminatory power" could be considered the most important guideline for signature choice, but this criterion has particular challenges. This can be described using a "chicken and egg" analogy: when testing discriminatory power, how do we know that two catchments have similar or different behaviour without using signatures to test it? We could approach this difficulty by comparing signature values with "soft" or "expert" knowledge of the similarities and differences between catchment behaviours. Alternatively, signature values could be analysed in experimental catchments where auxiliary data provide an independent source of information about the catchment behaviour, e.g. direct measurements of vertical water re-distribution to compare with FDC signature values.

More generally, when designing signatures for a specific catchment, we must think critically about factors that can impact the link between signature values and hydrological processes, using information about catchment configuration, spatial heterogeneity, human impacts, etc. For example, the FDC signatures considered in this paper might present difficulties for 
signature design, because the FDC shape combines the effects of multiple hydrological processes, for example, mid-range flow values could result from a combination of small flow peaks and late recession times from large flow peaks.

A secondary challenge that is not investigated in this commentary, but forms a companion question, is to discuss guidelines for selecting a "complete set" of signatures for future applications. This task relates to many of the signature selection statements reviewed in Section 2, such as an attempt to select signatures that are independent, or that cover a wide range of hydrological behaviours.

In conclusion, we have proposed five guidelines that could be used to evaluate potential formulations of hydrological signatures. Using the example of the FDC, we demonstrated how assessment against each guideline could be used to compare or choose between alternative signature definitions, depending on the intended application.

\section{Acknowledgements}

IW acknowledges the financial support of The Swedish Research Council Formas (9422015-321).

\section{References}

Coxon, G., Freer, J., Wagener, T., Odoni, N.A. and Clark, M., 2014. Diagnostic evaluation of multiple hypotheses of hydrological behaviour in a limits-of-acceptability framework for 24 UK catchments. Hydrological Processes, 28(25), pp.6135-6150.

Euser, T., Winsemius, H.C., Hrachowitz, M., Fenicia, F., Uhlenbrook, S. and Savenije, H.H.G., 2013. A framework to assess the realism of model structures using hydrological signatures. Hydrology and Earth System Sciences, 17 (5), 2013.

Gupta, H.V., Wagener, T. and Liu, Y., 2008. Reconciling theory with observations: elements of a diagnostic approach to model evaluation. Hydrological Processes, 22(18), pp.3802-3813.

Kirchner, J. W. (2016a). Aggregation in environmental systems-Part 2: Catchment mean transit times and young water fractions under hydrologic nonstationarity. Hydrology and Earth System Sciences, 20(1), 299-328.

Kirchner, J. W. (2016b). Aggregation in environmental systems-Part 1: Seasonal tracer cycles quantify young water fractions, but not mean transit times, in spatially heterogeneous catchments. Hydrology and Earth System Sciences, 20(1), 279-297.

McMillan, H. K. and Westerberg, I. K. 2015. Rating curve estimation under epistemic uncertainty. Hydrological Processes 29: 1873-1882. DOI: 10.1002/hyp.10419

Sawicz, K., Wagener, T., Sivapalan, M., Troch, P. A., and Carrillo, G., 2011. Catchment classification: empirical analysis of hydrologic similarity based on catchment function in the eastern USA, Hydrol. Earth Syst. Sci., 15, 2895-2911, doi:10.5194/hess-15-2895-2011.

Sawicz, K.A., Kelleher, C., Wagener, T., Troch, P., Sivapalan, M. and Carrillo, G., 2014. Characterizing hydrologic change through catchment classification. Hydrology and Earth System Sciences, 18(1), pp.273-285.

Schaefli, B., 2016. Snow hydrology signatures for model identification within a limits-ofacceptability approach. Hydrological Processes, 30(22), pp.4019-4035. 
Vogel, R.M. and Fennessey, N.M., 1994. Flow-duration curves. I: New interpretation and confidence intervals. Journal of Water Resources Planning and Management, 120(4), pp.485504.

Westerberg, I.K., Guerrero, J-L., Younger, P-M., Beven, K. J., Seibert, J., Halldin, S., J-E. Freer, Xu, C-Y. 2011. Calibration of hydrologic models using flow-duration curves. Hydrology and Earth System Sciences, 15, pp. 2205-2227.

Westerberg, I.K. and McMillan, H.K., 2015. Uncertainty in hydrological signatures. Hydrology and Earth System Sciences, 19(9), pp.3951-3968.

Westerberg, I.K., Wagener, T., Coxon, G., McMillan, H.K., Castellarin, A., Montanari, A. and Freer, J., 2016. Uncertainty in hydrological signatures for gauged and ungauged catchments. Water Resources Research, 52(3), pp.1847-1865.

Yadav, M., Wagener, T. and Gupta, H., 2007. Regionalization of constraints on expected watershed response behavior for improved predictions in ungauged basins. Advances in Water Resources, 30(8), pp.1756-1774.

Yilmaz, K. K., H. V. Gupta, and T. Wagener (2008), A process-based diagnostic approach to model evaluation: Application to the NWS distributed hydrologic model, Water Resour. Res., 44, W09417, doi:10.1029/2007WR006716.

Yokoo, Y. and Sivapalan, M., 2011. Towards reconstruction of the flow duration curve: development of a conceptual framework with a physical basis. Hydrology and Earth System Sciences, 15(9), pp.2805-2819. 